\title{
MicroRNA-124a and microRNA-34b/c are frequently methylated in all histological types of colorectal cancer and polyps, and in the adjacent normal mucosa
}

\author{
GUOREN DENG $^{1}$, SANJAY KAKAR ${ }^{2}$ and YOUNG S. KIM ${ }^{1}$ \\ Departments of ${ }^{1}$ Medicine and ${ }^{2}$ Pathology, Veterans Affairs Medical Center, \\ University of California at San Francisco, San Francisco, CA 94121, USA
}

Received August 8, 2010; Accepted November 15, 2010

DOI: $10.3892 / \mathrm{ol} .2010 .222$

\begin{abstract}
MicroRNAs (miRs) are a class of small RNAs that regulate gene expression at the post-transcriptional and/ or translational level by interacting with their target mRNAs. miRs are down-regulated or up-regulated in various cancer types, triggering abnormal cell differentiation, proliferation and apoptosis. miR-124a and miR-34b/c have been reported to be expressed at lower levels in colorectal cancer (CRC) due to methylation of these genes. The present study aimed to determine the methylation status of miR-124a and miR-34b/c in CRCs and polyps of various histological types, adjacent normal mucosa and ulcerative colitis. The colon cancer cell line study showed an association of the lower expression of miR-124a and miR-34b/c with the methylation of these genes and induction of the expression of these genes with the treatment by 5-aza-2'-deoxycytidine. Among nine different cancer types examined, CRC showed the highest frequency of methylation of miR-124a (cell lines $88 \%$ and tissues 99\%) and miR-34b/c (cell lines 89\% and tissues 93\%). Mucinous and non-mucinous CRCs and all the histological types of colorectal polyps showed a high frequency of methylation of miR-124a and miR-34b/c. Notably, methylation of miR-124a $(59 \%)$ and miR-34b/c (26\%) was observed in the adjacent normal mucosa of CRC patients, but not in colonic mucosa from patients without cancer or with ulcerative colitis. The methylation of miR-124a in the adjacent normal mucosa was associated with the microsatellite instability of CRC, while the methylation of $\mathrm{miR}-34 \mathrm{~b} / \mathrm{c}$ was associated with an older age at diagnosis of CRC. The results showed that the methylation of miR-124a and miR-34b/c occured early in colorectal carcinogenesis and certain CRCs may arise from a field defect defined by the epigenetic inactivation of miRs.
\end{abstract}

Correspondence to: Dr Guoren Deng, Department of Medicine, Veterans Affairs Medical Center, University of California at San Francisco, 4150 Clement Street, Mailbox 112F, San Francisco, CA 94121, USA

E-mail: guoren.deng@ucsf.edu

Key words: microRNA, epigenetic regulation, colorectal cancer

\section{Introduction}

MicroRNAs (miRs) are small, non-coding RNAs that regulate gene expression at the transcriptional, post-transcriptional and/ or translational level. Approximately $30 \%$ of human genes are estimated to be the targets of miRs, which play a role in diverse biological processes such as development, proliferation, differentiation and apoptosis $(1,2)$. Altered levels of the expression of miRs resulting from their deregulation occur in a wide range of human diseases, including cancer, where miRs act as either tumor suppressor genes or oncogenes and are involved in the initiation, progression and dissemination of cancer $(1,2)$. Aberrant regulation of miRs is caused by either genetic or epigenetic alterations, impairment in miR processing or both $(1,2)$. The genetic alteration of miRs is caused mainly by chromosomal abnormalities, since more than $50 \%$ of miR genes are located on fragile sites, although mutations occur (1). The epigenetic cause of miR deregulation involves silencing miR expression by $\mathrm{CpG}$ island methylation and repressive histone modifications (1). Defects in the miR processing machinery include altered levels of processing enzymes and defective activation of pathways that facilitate the processing $(1,2)$.

In colorectal carcinogenesis, epigenetic changes are common phenomena in pre-cancerous and cancerous lesions, in addition to the genomic changes that frequently occur in these lesions (3-7). Epigenetic changes may be found in the apparently normal colonic mucosa adjacent to cancer exhibiting similar changes, indicating a field effect $(8,9)$. Recently, an altered level of the expression of miRs has been reported in colorectal cancer (CRC) and certain miRs are expressed at low levels in CRC due to the hypermethylation of $\mathrm{miR}$ genes (10). To identify miRs with epigenetically silenced expression in CRC, colon cancer cells, with or without genetic deficiency for the DNA methyltransferase enzymes, were used in combination with miR expression profiling $(11,12)$. Among the epigenetically silenced miRs identified using these screening methods were miR-124a and miR-34b/c, which showed frequent epigenetic silencing in CRC tissues, but not in the adjacent normal mucosa, miR-124a (42/56, 75\%) and miR-34b/c (101/111, 90\%) (11,12).

The present study aimed to compare the frequency of the epigenetic silencing of miRNA-124a and miRNA-34b/c in 
Table I. Comparison of miR-124a and miR-34b/c expression levels with methylation status.

\begin{tabular}{|c|c|c|c|c|}
\hline & \multicolumn{2}{|c|}{ miR-124a } & \multicolumn{2}{|c|}{$\mathrm{miR}-34 \mathrm{~b} / \mathrm{c}$} \\
\hline & Expression & Methylation & Expression & Methylation \\
\hline $\mathrm{Caco} 2$ & 330.39 & - & nd & nd \\
\hline HCT116 & 1.65 & + & nd & nd \\
\hline RKO & 1.00 & + & 1.74 & + \\
\hline Colo201 & nd & nd & 1.00 & + \\
\hline Colo320 & 85.72 & - & 1.08 & + \\
\hline LS174T & nd & nd & 15.25 & - \\
\hline VACO5 & 1.58 & + & 1.50 & + \\
\hline
\end{tabular}

nd, not determined.

mucinous and non-mucinous CRCs, various histological types of colorectal polyps, uninvolved mucosa adjacent to CRC, normal mucosa from non-cancer bearing subjects and ulcerative colitis, as well as to compare the frequencies of miR expression in cell lines and cancer tissues from different organs.

\section{Materials and methods}

Cell lines and primary tumors. Colorectal cancer cell lines C, C1a, Caco2, Colo201, Colo320, H498, HCT8, HCT1116, HRT18, HT29, Lovo, LS123, LS174T, RKO, RW2982, SW48, SW620, SW1116, SW1463, T74, VACO5, VACO6, VACO10P, VACO411 and VACO703 were grown in DMEM, supplemented with $10 \%$ fetal bovine serum at $37^{\circ} \mathrm{C}$ in a $5 \% \mathrm{CO}_{2}$ atmosphere. Formalin-fixed, paraffin-embedded tissue blocks of primary CRCs, colorectal polyps, ulcerative colitis, normal mucosa and primary cancers from the pancreas, stomach, liver, lung, breast, kidney, prostate and melanomas were obtained from the Department of Pathology, University of California at San Francisco and the San Francisco Veterans Affairs Medical Center, USA. This study was approved by the institutional review boards of the institutions.

5-Aza-2'-deoxycytidine (5-aza-dC) treatment. Colo320, RKO, SW48 and VACO5 cells were grown in 6-well plates on day 0 and treated with $5 \mu \mathrm{M}$ 5-aza-dC (Sigma, St. Louis, MO, USA) on day 1 . The medium was replaced on day 2 . The cells were grown for 2 days and harvested on day 4 .

Measurement of the expression of miR-124a and miR-34b/c. Total small RNA was isolated from the cultured cells using a miRNeasy Mini kit (Qiagen, Valencia, CA, USA), according to the manufacturer's instructions. cDNAs were synthesized using specific miRNA primers (Applied Biosystems, Foster City, CA, USA), or using hexanucleotides mix and SuperScript first-strand cDNA synthesis kit (Invitrogen, Carlsbad, CA, USA). Gene expression was measured by quantitative real-time PCR with Applied Biosystem 7500 Fast Sequence Detection and Taq Man assay kits (Applied Biosystems), or by regular PCR using the primers: 5'-TGAGGGCCCCTCTGCGTGT (miR-124a, forward) and 5'-AGGCGCCTCTCTTGGCATT (miR-124a, reverse); or 5'-GTGCTCGGTTTGTAGGCAGT
(miR-34b/c, forward) and 5'-GTGCCTTGTTTTGATGGCAG (miR-34b/c, reverse).

Methylationanalysis.DNA obtained from the celllines or tissues were modified with $\mathrm{NaHSO}_{3}$ (3). The DNA methylation status of miR-124a and miR-34b/c was determined by methylationspecific PCR (MSP). For miR-124a, the $\mathrm{NaHSO}_{3}$-modified DNA was amplified separately by PCR with either methylationspecific primers, i.e., 5'-GCGAGGATTTTACGTAAGTTC (forward) and 5'-CAAAAAAACCCTCAAAACTAAAAC GAACG (reverse), or unmethylation-specific primers, i.e., 5'-TGGGTGAGGATTTTATGTAAGTTT (forward) and 5'-CACA AAAAAACCCTCAAA ACTAAAACAAACA (reverse). For miR-34b/c, the modified DNAs were amplified by PCR with either methylation-specific primers, i.e, 5'-CGGTGAAATGGGGTTCGAGGC (forward) and 5'-CCGAACACCGAACACCCGCG(reverse),orunmethylationspecific primers, i.e., 5'-TGTTTTTTGGTGAAATGGGG TTTGAGGT (forward) and 5'-CCTACAAACCAAACAC CAAACACCCACA (reverse).

Analysis of microsatellite instability (MSI), CpG island methylation phenotype (CIMP), loss of heterozygosity ( $\mathrm{LOH})$ and BRAF, KRAS and p53 mutations. DNA was isolated from the microdissected samples and the analysis of MSI, CIMP, LOH and BRAF, KRAS and p53 gene mutations was performed as previously described (13).

Statistical analysis. A comparison of the categorical variables was performed using the Chi-square and Fisher's exact tests. The Student's t-test was used for comparison of the mean age of the patients. $\mathrm{P}<0.05$ was considered to be statistically significant.

\section{Results}

Correlation of the expression levels of $m i R-124 a$ and $m i R$ $34 \mathrm{~b} / \mathrm{c}$ with the methylation status in colon cancer cell lines. The expression levels of miR-124a and miR-34b/c, quantified by real-time PCR using 124a- and miR-34b/c-specific primers, showed a positive correlation with their methylation status (Table I). MiR-124a was expressed at significantly lower levels 

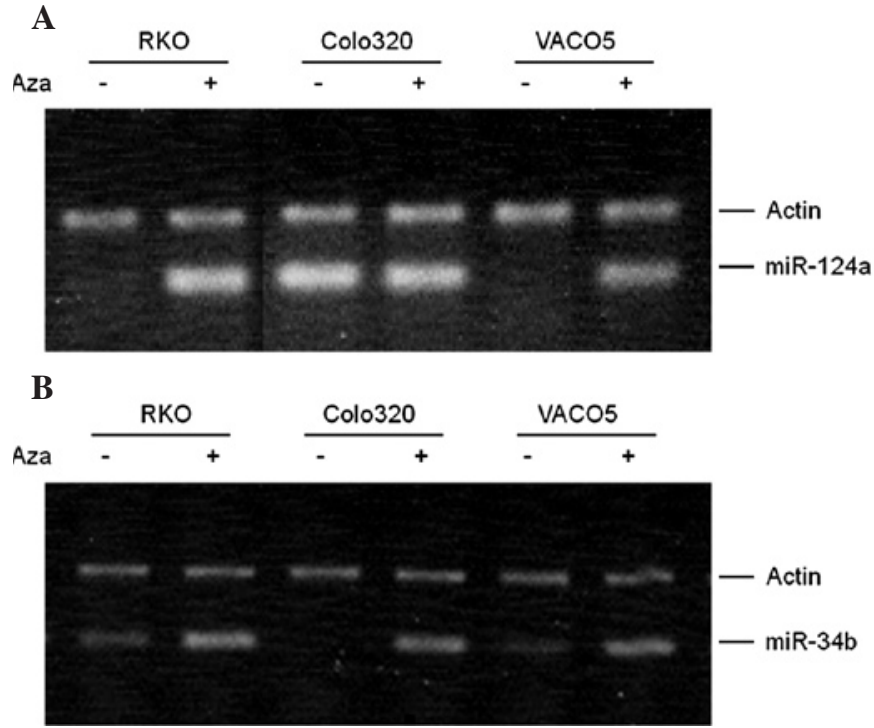

Figure 1. miR-124a and miR-34b/c expression in colorectal cancer cell lines RKO, Colo320 and VACO5, before (-) and after (+) the 5-aza-dC treatment. RNA was isolated from the cells and the expression of (A) miR-124a and (B miR-34b/c was measured by RT-PCR.

in HCT116, RKO and VACO5 cells compared to Caco2 and Colo320 cells, concomitant with methylation in HCT116, RKO and VACO5 cells, but not in Caco2 and Colo320 cells. The low expression level of miR-34b/c also correlated with methylation (Table I). The cell lines with a significantly lower expression of miR-34b/c (RKO, Colo201, Colo320 and VACO5) showed methylation, while LS174 cells which showed higher levels of miR-34b/c expression did not show methylation of miR-34b/c. Methylation of these miR genes is a common event in colon cancer cells; $88 \%(22 / 25)$ of colon cancer cell lines showed methylation of miR-124a and 89\% (17/19) showed methylated miR-34b/c.

Expression of miR-124a and miR-34b was induced by the 5-aza-dC treatment. RKO, Colo320 and VACO5 cells were treated with 5-aza-dC and the expression of miR-124a and miR-34b/c was measured by RT-PCR (Fig. 1). RKO and
VACO5 cells, which had no detectable miR-124a, showed a significant induction of miR-124a following treatment, indicating that miR-124a was methylated, while Colo320, which had a high-level expression of miR-124a, did not show induction, indicating that miR-124a was unmethylated. After the 5-aza-dC treatment, the three cell lines with either low (RKO and VACO5) or undetectable (Colo320) levels of miR34b/c expression showed significant induction of miR-34b/c, indicating that miR-34b/c is methylated at a moderate to high level in these cells.

Methylation of miR-124a and miR-34b is frequently present in colorectal cancers and colonic polyps. The methylation status of miR-124a and miR-34b was determined in the primary tumors of the colorectum, stomach, pancreas, liver, lung, breast, kidney, prostate and melanomas (Table II). miR-124a was most frequently methylated in CRC $(80 / 81,99 \%)$ and prostate cancer $(4 / 4,100 \%)$ compared to other cancer types whose frequency ranged from 17 to $67 \%$. Hepatocellular carcinoma showed no methylation of miR-124a (0/9, 0\%). Methylation of miR-34b/c was also most frequently observed in CRC (63/68, $93 \%$ ), compared to other cancer types which showed methylation frequencies ranging from 17 to $69 \%$ (Table II). Mucinous and non-mucinous CRCs showed similar methylation frequencies of miR-124a and miR-34b/c (Table III). The methylation of both miR-124a and miR-34b/c were observed frequently in all histological types of colonic polyps $(121 / 131,92 \%$ for miR-124a; 145/152, 95\% for miR-34b/c) (Table III). This result indicates that the methylation of miR-124a and miR-34b/c is an early event in colorectal carcinogenesis.

Methylation of $m i R-124 a$ and $m i R-34 b$ was present in normal mucosa adjacent to colorectal cancers. Normal mucosa adjacent to CRC was microdissected and the methylation status of miR-124a and miR-34b was analyzed (Table III). Methylation of miR-124a was observed in the adjacent mucosa in 59\% of cases (29/49), compared to $26 \%$ for miR-34b/c (12/46) (Table III). By contrast, none of the colonic mucosa from the subjects without CRC showed methylation of miR-124a (0/12) or of miR-34b/c (0/19). In addition, none of the ulcerative colitis specimens showed methylation of miR-124a $(0 / 20)$ or miR-34b/c (0/24) (Table III).

Table II. Methylation of miR-124a and miR-34b/c in primary tumors.

\begin{tabular}{|c|c|c|c|c|c|c|}
\hline & \multicolumn{3}{|c|}{ miR-124a } & \multicolumn{3}{|c|}{$\mathrm{miR}-34 \mathrm{~b} / \mathrm{c}$} \\
\hline & Total & Methylated & $\%$ & Total & Methylated & $\%$ \\
\hline Colorectum & 81 & 80 & 99 & 68 & 63 & 93 \\
\hline Pancreas & 9 & 5 & 56 & 10 & 5 & 50 \\
\hline Stomach & 15 & 10 & 67 & 41 & 31 & 69 \\
\hline Liver & 9 & 0 & 0 & 9 & 2 & 22 \\
\hline Lung & 9 & 3 & 33 & 7 & 2 & 29 \\
\hline Breast & 24 & 4 & 17 & 40 & 22 & 55 \\
\hline Kidney & 8 & 2 & 25 & 10 & 4 & 40 \\
\hline Prostate & 4 & 4 & 100 & 5 & 3 & 60 \\
\hline Melanoma & 5 & 3 & 60 & 6 & 1 & 17 \\
\hline
\end{tabular}


Table III. Methylation of miR-124a and $\mathrm{miR}-34 \mathrm{~b} / \mathrm{c}$ in colorectal tissues.

\begin{tabular}{|c|c|c|c|c|c|c|}
\hline \multirow[t]{2}{*}{ Colorectal tissue } & \multicolumn{3}{|c|}{ miR-124a } & \multicolumn{3}{|c|}{$\mathrm{miR}-34 \mathrm{~b} / \mathrm{c}$} \\
\hline & Total & Methylated & $\%$ & Total & Methylated & $\%$ \\
\hline Normal mucosa & 12 & 0 & 0 & 19 & 0 & 0 \\
\hline Ulcerative colitis & 20 & 0 & 0 & 24 & 0 & 0 \\
\hline Colonic polyps & 131 & 121 & 92 & 152 & 145 & 95 \\
\hline $\mathrm{HP}$ & 38 & 35 & 92 & 42 & 42 & 100 \\
\hline SSA & 25 & 24 & 96 & 27 & 26 & 96 \\
\hline SA & 23 & 22 & 96 & 24 & 23 & 96 \\
\hline TVA & 22 & 20 & 90 & 31 & 30 & 97 \\
\hline $\mathrm{TA}$ & 23 & 20 & 87 & 28 & 24 & 86 \\
\hline Mucosa adjacent to cancer & 49 & 29 & 59 & 46 & 12 & 26 \\
\hline Cancer & 81 & 80 & 99 & 68 & 63 & 93 \\
\hline $\mathrm{MC}$ & 10 & 10 & 100 & 14 & 12 & 86 \\
\hline NMC & 71 & 70 & 99 & 54 & 51 & 94 \\
\hline
\end{tabular}

HP, hyperplastic polyp; SSA, sessile serrated adenoma; SA, serrated adenoma; TVA, tubulovillous adenoma; TA, tubular adenoma; MC, mucinous cancer; NMC, non-mucinous cancer.

Correlation of the methylation status of miR-124a and miR$34 \mathrm{~b} / \mathrm{c}$ in CRC and adjacent normal mucosa with clinical and molecular charateristics of CRC. When the presence of miR124a methylation in the adjacent apparently normal colonic mucosa was compared to the clinical and molecular characteristics of CRC, a significant correlation was observed between the methylation of miR-124a in the adjacent mucosa and CIMP $(\mathrm{p}<0.001)$ (Table IV). A borderline correlation was observed between miR-124a methylation and proximal tumor location $(p<0.081)$, MSI $(p<0.070)$ and BRAF mutations $(p<0.069)$. By contrast, the methylation of $\mathrm{miR}-34 \mathrm{~b} / \mathrm{c}$ in the adjacent colonic mucosa was significantly correlated with patient age $(\mathrm{p}<0.004)$, but no correlation was detected with other clinical and molecular characteristics of CRC (Table IV).

\section{Discussion}

The present study showed that miR-124a and miR-34b/c are most frequently methylated in CRC compared to eight other tumor types, indicating a relative colon tissue typespecific expression of these miRs. We observed that the two miRs are frequently methylated in colon cancer cell lines (miR-124a, 22/25, 88\% and miR-34b/c, 17/19, 89\%) and that methylation is correlated with the low-level expression of these miRs. Lujambio et al have reported that HCT116 colon cancer cells that express low levels of miR-124a, showed a 2- to 3-fold up-regulation of miR-124a expression, following treatment with the demethylating agent 5-aza-dC, or when made genetically deficient for the DNA methylatransferase enzymes (11). The data indicate that DNA methylationassociated repression of miR-124a occurs frequently in colon cancer cells. A similar result was observed with miR-34b/c in HCT116 cells (12). Epigenetic loss of miR-124a expression was found to be associated with the activation of an oncogene, cyclin $\mathrm{D}$ kinase 6 , and the phosphorylation of the retino- blastoma tumor suppressor gene, and that $\mathrm{miR}-34 \mathrm{~b} / \mathrm{c}$ are targets of p53, indicating the functional significance of the epigenetic regulation of these genes $(11,12,14)$. We observed that RKO and VACO5 colon cancer cells exhibited no detectable levels of miR-124a, and that RKO, VACO5 and Colo320 colon cancer cells exhibited low or undetectable levels of miR-34b/c, which showed a significant induction of the respective miRs, following 5 -aza-dC treatment, indicating that the epigenetic silencing of these miRs occurs in colon cancer cells. These observations are consistent with previous reports (10-12).

No detectable methylation of miR-124a or miR-34b/c was noted in the normal colonic tissues from the subjects without cancer, or in the colonic mucosa of patients with ulcerative colitis. However, frequent methylation of miR-124a (59\%) and miR-34b/c (26\%) was noted in the apparently normal mucosa adjacent to the CRCs with methylation of these genes, indicating a field effect or field cancerization $(8,15,16)$. We have recently reported that epigenetic silencing of oncostatin $\mathrm{M}$ receptor (OSMR) occurs in 55\% of apparently normal mucosa adjacent to CRCs (9). Together, these data indicate that epigenetic regulation of OSMR, miR-124a and miR-34b/c may function as mediators of the field effect or field cancerization. We previously reported that different histological types of colonic polyps (serrated vs. tubular adenoma) and colon cancer (mucinous vs. non-mucinous) show different frequencies and patterns of $\mathrm{CpG}$ island methylation of various genes $(17,18)$. However, in the present study, high frequencies of methylation of miR-124a and miR-34b/c were observed in all histological types of colonic polyps and CRC. Together with the data regarding adjacent normal mucosa, our data on colonic polyps indicate that methylation of miR-124a and miR-34b/c occurs early in multi-step colorectal carcinogenesis.

The genetic instability pathways, chromosomal instability (CIN) and MSI, as well as additional pathways involving epige- 
Table IV. Correlation of methylation status of miR-124a and miR-34b/c in colorectal cancer and adjacent normal mucosa with clinical and molecular characteristics in cancer.

\begin{tabular}{|c|c|c|c|c|c|c|}
\hline & \multicolumn{3}{|c|}{ miR-124a (adjacent/cancer) } & \multicolumn{3}{|c|}{ miR-34b/c (adjacent/cancer) } \\
\hline & $+/+$ & $-/+$ & p-value & $+/+$ & $-/+$ & p-value \\
\hline Total & 29 & 20 & & 12 & 34 & \\
\hline Age & & & ns & & & 0.004 \\
\hline Mean & 69.0 & 68.2 & & 76.8 & 66.4 & \\
\hline $\mathrm{SD}$ & 12.1 & 15.2 & & 7.7 & 14.6 & \\
\hline Gender & & & ns & & & ns \\
\hline Male & $14(54 \%)$ & $12(46 \%)$ & & $7(29 \%)$ & $17(71 \%)$ & \\
\hline Female & $14(64 \%)$ & $8(36 \%)$ & & $5(24 \%)$ & $16(76 \%)$ & \\
\hline Stage & & & ns & & & ns \\
\hline $\mathrm{A} / \mathrm{B}$ & $17(57 \%)$ & $13(43 \%)$ & & $6(21 \%)$ & $23(79 \%)$ & \\
\hline $\mathrm{C} / \mathrm{D}$ & $12(63 \%)$ & $7(37 \%)$ & & $6(35 \%)$ & $11(65 \%)$ & \\
\hline Tumor side & & & 0.081 & & & $\mathrm{~ns}$ \\
\hline Proximal & $15(75 \%)$ & $5(25 \%)$ & & $5(26 \%)$ & $14(74 \%)$ & \\
\hline Distal & $14(48 \%)$ & $15(52 \%)$ & & $7(26 \%)$ & $20(74 \%)$ & \\
\hline Survival (5 years) & & & ns & & & $\mathrm{ns}$ \\
\hline- & $16(70 \%)$ & $7(30 \%)$ & & $7(32 \%)$ & $15(68 \%)$ & \\
\hline+ & $13(50 \%)$ & $13(50 \%)$ & & $5(21 \%)$ & $19(79 \%)$ & \\
\hline MSI & & & 0.070 & & & ns \\
\hline+ & $5(100 \%)$ & $0(0 \%)$ & & $0(0 \%)$ & $5(100 \%)$ & \\
\hline- & $24(55 \%)$ & $20(45 \%)$ & & $12(29 \%)$ & $29(71 \%)$ & \\
\hline $\operatorname{CIMP}(\geq 3 / 6)$ & & & $<0.001$ & & & $\mathrm{~ns}$ \\
\hline+ & $13(100 \%)$ & $0(0 \%)$ & & $3(23 \%)$ & $10(77 \%)$ & \\
\hline- & $16(44 \%)$ & $20(56 \%)$ & & $9(27 \%)$ & $24(73 \%)$ & \\
\hline $\mathrm{LOH}(\geq 1 / 4)$ & & & ns & & & ns \\
\hline+ & $21(55 \%)$ & $17(45 \%)$ & & $11(30 \%)$ & $26(70 \%)$ & \\
\hline- & $8(73 \%)$ & $3(27 \%)$ & & $1(11 \%)$ & $8(89 \%)$ & \\
\hline BRAF mutations & & & 0.069 & & & ns \\
\hline+ & $6(100 \%)$ & $0(0 \%)$ & & $1(14 \%)$ & $6(86 \%)$ & \\
\hline- & $23(53 \%)$ & $20(47 \%)$ & & $11(28 \%)$ & $28(72 \%)$ & \\
\hline KRAS mutations & & & ns & & & ns \\
\hline+ & $7(54 \%)$ & $6(46 \%)$ & & $3(21 \%)$ & $11(79 \%)$ & \\
\hline- & $22(61 \%)$ & $14(39 \%)$ & & $9(28 \%)$ & $23(72 \%)$ & \\
\hline p53 mutations & & & ns & & & ns \\
\hline+ & $16(57 \%)$ & $12(43 \%)$ & & $6(24 \%)$ & $19(76 \%)$ & \\
\hline- & $13(62 \%)$ & $8(38 \%)$ & & $6(29 \%)$ & $15(71 \%)$ & \\
\hline
\end{tabular}

+/+, methylation is present in apparently adjacent normal colonic mucosa and CRC; -/+, methylation is present in CRC, but not in adjacent mucosa; SD, standard deviation; ns, not significant; MSI, microsatellite instability; CIMP, CpG island methylation phenotype; LOH, loss of heterozygosity.

netic abnormalities, are known to be involved in colorectal carcinogenesis $(4-6,19)$. CRCs with CIN are characterized by frequent LOH, KRAS and p53 mutations, whereas CRCs with MSI frequently show a high degree of concurrent promoter methylation in multiple genes, i.e., CIMP, and BRAF mutations (5-7,19). CRCs with MSI show different clinicopathological characteristics from CIN tumors, such as a younger age at diagnosis, proximal location, presence of tumor-infiltrating lymphocytes and mucinous histology $(5-7,19)$. The potential relationship of the methylation status of miR-124a and miR$34 \mathrm{~b} / \mathrm{c}$ of CRCs and apparently normal adjacent mucosa to these molecular and clinicopathological characteristics was examined. We noted a significant correlation of the methylation of miR-124a in the apparently adjacent normal mucosa to CIMP, and a borderline correlation with proximal tumor location, MSI and BRAF mutations, molecular characteristics frequently found in CRCs with MSI. By contrast, the methylation of $\mathrm{miR}-34 \mathrm{~b} / \mathrm{c}$ showed a significant correlation only with 
older age at diagnosis. These findings are significant since promoter methylation in CRCs was broadly classified into cancer- and age-associated types $(5,6,20)$.

In conclusion, we confirmed the frequent occurrence of epigenetic silencing of miR-124a and miR-34b/c in CRCs. We also noted that the methylation of the two miRs occurs frequently in apparently normal adjacent colonic mucosa, indicating a field effect or field cancerization; that mucinous and non-mucinuous CRCs and all histological types of colonic polyps show frequent methylation of the two miRs, indicating that methylation of these miRs is an early event in all pathways of colorectal carcinogenesis; and finally, that the methylation of miR-124a in the apparently adjacent normal colonic mucosa is associated with MSI of CRCs, while the methylation of miR-34b/c is associated with older age at diagnosis, underscoring the importance of the relationship between promoter methylation, cancer and aging.

\section{Acknowledgements}

This study was supported in part by the Department of Veterans Affairs Medical Research Service, the Oberkotter Foundation grant and the Theodora Betz Foundation grant.

\section{References}

1. Croce CM: Causes and consequences of microRNA dysregulation in cancer. Nat Rev Genet 10: 704-714, 2009.

2. Davalos V and Esteller M: MicroRNAs and cancer epigenetics: a macrorevolution. Curr Opin Oncol 22: 35-45, 2010.

3. Vogelstein B and Kinzler KW: Cancer genes and the pathways they control. Nat Med 10: 789-799, 2004.

4. Jones PA and Baylin SB: The epigenomics of cancer. Cell 128: 683-692, 2007.

5. Issa JP: CpG island methylator phenotype in cancer. Nat Rev Cancer 4: 988-993, 2004.

6. Kim YS and Deng G: Epigenetic changes (aberrant DNA methylation) in colorectal neoplasia. Gut Liver 1: 12-21, 2007.
7. Deng G, Nguyen A, Tanaka H, et al: Regional hypermethylation and global hypomethylation are associated with altered chromatin conformation and histone acetylation in colorectal cancer. Int J Cancer 118: 2999-3005, 2006.

8. Shen L, Kondo Y, Rosner GL, et al: MGMT promoter methylation and field defect in sporadic colorectal cancer. J Natl Cancer Inst 97: 1330-1338, 2005.

9. Deng G, Karkar S, Okudaira K, Choi E, Sleisenger MH and Kim YS: Unique methylation pattern of oncostatin M receptor gene in cancer of colorectum and other digestive organs. Clin Cancer Res 15: 1519-1526, 2009.

10. Faber C, Kirchner T and Hlubeck F: The impact of microRNAs in colorectal cancer. Virchows Arch 454: 359-367, 2009.

11. Lujambio A, Ropero S, Ballestar E, et al: Genetic unmasking of an epigenetically silenced microRNA in human cancer cells. Cancer Res 67: 1424-1429, 2007.

12. Toyota M, Suzuki H, Sasakin Y, et al: Epigenetic silencing of microRNA-34b/c and B-cell translocation gene 4 is associated with $\mathrm{CpG}$ island methylation in colorectal cancer. Cancer Res 68: 4123-4132, 2008.

13. Deng G, Kakar S, Tanaka H, Matsuzaki K, Miura S, Sleisenger MH and Kim YS: Proximal and distal colorectal cancers show distinct gene-specific methylation profile and clinical and molecular characteristics. Eur J Cancer 44: 1290-1301, 2008.

14. He L, He X, Lowe SW and Hannon GJ: microRNAs join the p53 network - another piece in the tumor suppressor puzzle. Nat Rev Cancer 7: 819-822, 2007.

15. Braakhuis BJ, Tabor MP, Kummer JA, Leemans CR and Brakenhoff RH: A genetic explanation of Slaughter's concept of field cancerization: evidence of clinical implication. Cancer Res 63: 1727-1730, 2003.

16. Leedham SJ, Graham TA, Oukuf D, et al: Clonality, founder mutations, and field cancerization in human ulcerative colitisassociated neoplasia. Gastroenterology 136: 542-550, 2009.

17. Kim YH, Kakar S, Cun L, Deng G and Kim YS: Distinct CpG island methylation profile and BRAF mutation status in serrated and adenomatous colorectal polyps. Int J Cancer 123: 2587-2593, 2008.

18. Tanaka H, Deng G, Matsuzaki K, et al: BRAF mutation, $\mathrm{CpG}$ island methylator phenotype and microsatellite instability occur more frequently and concordantly in mucinous than nonmucinous colorectal cancer. Int J Cancer 118: 2765-2771, 2005.

19. Wong JJL, Hawkins NJ and Ward RL: Colorectal cancer: a model for epigenetic tumorigenesis. Gut 56: 140-148, 2007.

20. Ahuja N, Li Q, Mohan AL, Baylin SB and Issa JP: Aging and DNA methylation in colorectal mucosa and cancer. Cancer Res 58: 5489-5494, 1998. 